\title{
QUANTIFICAÇÃO DE DEXTRANAS EM AÇÚCARES E EM CACHAÇAS
}

Manoel Gabriel Rodrigues Filho, Alexandre Ferreira Leite Neto, Francisco Wendel B. Aquino, Ana Maria G. Plepis, Ubirajara P. Rodrigues-Filho e Douglas Wagner Franco*

Instituto de Química de São Carlos, Universidade de São Paulo, Av. Trabalhador São Carlense, 400, 13560-970 São Carlos - SP, Brasil

Recebido em 4/5/06; aceito em 14/11/06; publicado na web em 28/5/07

\begin{abstract}
QUANTIFICATION OF DEXTRANS IN SUGARS AND IN BRAZILIAN SUGAR-CANE SPIRITS. Solid dextrans are thermally stable polysaccharides losing water only at $160^{\circ} \mathrm{C}$. According to IR, X-ray, DTA and DSC data no noticeable changes in dextran configuration occurs at this temperature. The total content of dextrans analyzed in 26 samples of Brazilian sugars and 57 samples of sweetened cachaças ranged from 109.5 to $1840 \mathrm{mg} / \mathrm{kg}$ and 1.6 to $11.2 \mathrm{mg} / \mathrm{L}$ with medians of $999.8 \mathrm{mg} / \mathrm{kg}$ and $5.9 \mathrm{mg} / \mathrm{L}$ respectively. Samples of sweeted cachaças have been monitored for turbidity, total soluble dextran content and weight of precipitate formed during 275 days. Precipitate formation is a kinetically controlled process which ends after 275 days when the total concentration of soluble dextrans becomes smaller than $0.25 \mathrm{mg} / \mathrm{L}$.
\end{abstract}

Keywords: dextrans; cachaça; flocculation.

\section{INTRODUÇÃO}

Para os produtores de açúcar a presença das dextranas é o principal indicador do grau de deterioração da cana pela ação da bactéria Leuconostoc mesenteroides ${ }^{1,2}$. Esta deterioração ocorre em função de fatores ambientais e de processamento, tais como idade da cana, variações abruptas de temperatura nas plantações, umidade no período da colheita e do tempo decorrido do corte até a moagem ${ }^{1,2}$.

As dextranas estão constantemente associadas aos problemas operacionais em usinas de açúcar e indústrias alimentícias, como o aumento da viscosidade dos xaropes e concentrados, o entupimento de filtros e o alongamento dos cristais de açúcar. Nas indústrias de bebidas é bem conhecido que as dextranas podem conduzir à formação de precipitados e alterações na estabilidade do produto acabado ${ }^{3-5}$.

A legislação vigente para a cachaça ${ }^{6}$ permite a adição de até 6,0 g/L de sacarose, sem a necessidade de qualquer indicação no rótulo. A partir de 6,0 até $30,0 \mathrm{~g} / \mathrm{L}$, torna-se obrigatória a inserção da expressão "cachaça adoçada" no rótulo do produto.

Para a cachaça adoçada, o principal problema relacionado à presença de dextranas é a formação de flocos. Estes apesar de atóxicos, são indesejáveis sob o ponto de vista comercial podendo, inclusive, conduzir ao descarte do produto ${ }^{7}$.

Procurando contribuir para o esclarecimento da presença de flocos em aguardentes, o presente trabalho descreve a aplicação do método de Robert's ${ }^{8}$ na determinação de dextranas totais em açúcares e em cachaças. A formação de flocos em aguardentes adoçadas é acompanhada em função das variações na concentração de dextranas totais, massa de precipitado acumulado e turbidez das amostras. A influência da temperatura de secagem das dextranas para a elaboração de soluções padrão é também discutida.

\section{PARTE EXPERIMENTAL}

\section{Instrumentação e reagentes}

As determinações espectrofotométricas foram executadas em

*e-mail: douglas@iqsc.usp.br espectrômetro Perkin-Elmer modelo Lambda 400. As curvas termogravimétricas foram obtidas utilizando-se uma balança termogravimétrica Perkin-Elmer TA-50 WSI. Para os experimentos de calorimetria exploratória diferencial (DSC) empregou-se um equipamento DSC-2010 da TA Instruments. Os difratogramas foram obtidos em difratômetro de raios-X de pó Siemens-D 5000.

Os padrões de dextranas $\mathrm{T}_{10}, \mathrm{~T}_{40}, \mathrm{~T}_{50}, \mathrm{~T}_{70}, \mathrm{~T}_{500}$ e $\mathrm{T}_{2000}$, indicando uma variação de massa molecular média de 1 × $10^{3}$ a $2 \times 10^{6} \mathrm{Da}$, foram adquiridos da Sigma-Aldrich.

Todas as soluções empregadas nas análises de dextranas totais pelo método de Robert's foram preparadas de acordo com o descrito na metodologia .

A quantificação de dextranas foi realizada em 26 amostras de açúcar comercial e em cachaças adoçadas (46 industriais e 11 artesanais) procedentes de várias regiões do Brasil.

\section{Metodologia}

Apesar de inespecífico com relação às características estruturais das dextranas, devido a sua simplicidade, o método descrito por Roberts ${ }^{8,9}$ é muito popular em laboratórios de controle de qualidade de indústrias que adicionam açúcar em seus produtos. Seguindo esta metodologia ${ }^{8-10}$, o preparo da curva de calibração foi efetuado com a secagem de $1,000 \mathrm{~g}$ dos padrões de dextranas $\left(\mathrm{T}_{10}\right.$, $\mathrm{T}_{40}, \mathrm{~T}_{50}, \mathrm{~T}_{70}, \mathrm{~T}_{500}, \mathrm{~T}_{2000}$ ) por $4 \mathrm{~h}$ a $105{ }^{\circ} \mathrm{C}$. Estes em seguida foram transferidos $(0,500 \mathrm{~g})$ para balões volumétricos de $500 \mathrm{~mL}$ onde foram dissolvidos com água tipo Milli-Q. As demais soluções foram obtidas a partir destas, por meio de diluição.

A reação de desenvolvimento colorimétrico utilizada na construção da curva de calibração e na quantificação das dextranas isoladas das amostras foi realizada em tubos de ensaio, misturando-se 2,0 mL dos padrões de dextranas (4,0 mL para amostras) com 1,0 $\mathrm{mL}$ de solução de fenol $5 \%$ e $10,0 \mathrm{~mL}$ de ácido sulfúrico concentrado. Em seguida os tubos foram imersos em água a $100{ }^{\circ} \mathrm{C}$ por 2 min, e então foram resfriados até a temperatura ambiente (15 a 20 min), efetuando-se as leituras de absorbância a $485 \mathrm{~nm}^{8-10}$.

$\mathrm{Na}$ determinação do teor de dextranas totais nas cachaças foram tomadas alíquotas de 10,0 $\mathrm{mL}$, nas quais adicionaram-se 40,0 
$\mathrm{mL}$ de etanol absoluto e $0,4 \mathrm{~g}$ de celite para a precipitação de todos os polissacarídeos. Estes foram separados por filtração em um funil com placa de vidro sinterizado ( $\varnothing$ de poros de 40 a $60 \mu \mathrm{m}$ ).

$\mathrm{O}$ precipitado retido no funil foi transferido quantitativamente para um balão de $25 \mathrm{~mL}$, que teve seu volume ajustado com água. Posteriormente, todo o volume do balão foi filtrado em papel de filtro Whatman no 42.

Uma alíquota de $10 \mathrm{~mL}$ do filtrado foi transferida para um tubo de ensaio e a esta foram adicionados $2 \mathrm{~mL}$ de $\mathrm{NaOH}$ 1,25 Mol/L, 2 $\mathrm{mL}$ da solução reagente de cobre e $0,2 \mathrm{~g}$ de celite. Após ser agitado, o tubo de ensaio foi aquecido em água (ebulição) por $5 \mathrm{~min}$ para precipitação do complexo $\mathrm{Cu}$-dextrana formado.

Depois de atingir a temperatura ambiente, o complexo foi filtrado em funil com placa de vidro sinterizado $(\varnothing$ de poros de 40 a $60 \mu \mathrm{m})$ e imediatamente lavado com duas porções de $10 \mathrm{~mL}$ de solução de lavagem. O filtrado foi descartado e o precipitado redissolvido por adição de 2,0 $\mathrm{mL}$ de ácido sulfúrico $1,0 \mathrm{~mol} / \mathrm{L} \mathrm{e}$ 2,0 mL de água. Os 4,0 mL resultantes da dissolução foram recolhidos em um tubo de ensaio e submetidos à reação colorimétrica.

Para as análises de dextranas totais nas amostras de açúcar, a mesma rotina analítica foi empregada, diferindo apenas na tomada inicial de amostra que foi de $20 \mathrm{~g}$ de açúcar dissolvidos com água em balão volumétrico de $50 \mathrm{~mL}$.

Para avaliação de reprodutibilidade, 10 réplicas das amostras (1 de cachaça e 1 de açúcar) contendo a mesma concentração de dextranas foram preparadas e cada medida foi realizada em duplicata. O limite de detecção (LD) foi estimado por diluições sucessivas de uma solução contendo concentração conhecida de dextranas até que o valor da absorbância medida correspondesse a três vezes o valor do ruído ${ }^{11}$.

As análises turbidimétricas foram realizadas utilizando-se um turbidímetro Hach (modelo 21700). A calibração foi efetuada com um conjunto de padrões entre 0 e 70 NTU, preparados por diluição de uma solução-estoque $(5,00 \mathrm{~mL}$ de solução de sulfato de hidrazina $0,01 \mathrm{mg} \mathrm{mL}^{-1}$ e $5,00 \mathrm{~mL}$ da solução hexametilenotetramina $0,10 \mathrm{mg}$ $\mathrm{mL}^{-1}$ ) em um volume de $100 \mathrm{~mL}$.

Nos experimentos turbidimétricos, de determinação de massa de precipitado e teor de dextranas totais, amostras de aguardentes adoçadas eram estocadas à temperatura ambiente e ao abrigo da luz. Nos diversos tempos indicados, as análises de turbidez foram realizadas. Em seguida, a amostra era submetida à filtração (membrana Milipore-ME $24 \varnothing$ de poro de $0,2 \mu \mathrm{m}$ e $\varnothing 47 \mathrm{~mm}$ ), efetuando-se no filtrado as análises de dextranas totais pelo método de Robert's, conforme descrito anteriormente. Por diferença de peso da membrana previamente tarada e após a sua secagem foi determinada a massa de precipitado formado.

Nos experimentos termogravimétricos, 6 a $8 \mathrm{mg}$ de amostras foram aquecidas de 20 a $600{ }^{\circ} \mathrm{C}$ a uma razão de aquecimento de 20 ${ }^{\circ} \mathrm{C} \min ^{-1}$, sob atmosfera de ar sintético e de nitrogênio, respectiva-

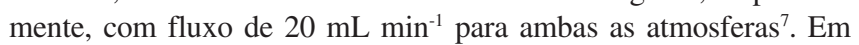
atmosfera de ar sintético também foram realizados os testes isotérmicos a 105 e $160{ }^{\circ} \mathrm{C}^{7}$.

Nas análises calorimétricas (DSC), 8 a $9 \mathrm{mg}$ de amostras foram aquecidas de 20 a $200{ }^{\circ} \mathrm{C}$ na presença de nitrogênio com fluxo de $20 \mathrm{~mL} \mathrm{~min}^{-1}$, com $20{ }^{\circ} \mathrm{C} \mathrm{min}^{-1}$ de razão de aquecimento ${ }^{7}$.

As análises de difração de raio-X foram conduzidas para 1,0 g das amostras $\mathrm{T}_{40}$ e $\mathrm{T}_{2000}$ nas seguintes condições experimentais: passo de $0,33^{\circ} \mathrm{s}^{-1}$, tempo de exposição de $1 \mathrm{~s}$ em módulo de varredura contínuo, com monocromador secundário de grafite e fendas de divergência com antiespalhamento de $3^{\circ}$, fenda variável do detector de 0,6 mm, tubo de raios- $\mathrm{X}$ de $\mathrm{Cu}$, a uma tensão de $40 \mathrm{kV}$ e uma corrente de $40 \mathrm{~mA}$. A radiação utilizada foi $\mathrm{Cu} \mathrm{K \alpha}=1,54056 \AA$ e o tempo de exposição foi de $5 \mathrm{~h}^{12}$.

\section{RESULTADOS E DISCUSSÃO}

De acordo com a metodologia original ${ }^{8,10}$ os padrões de dextranas devem ser secos a $105^{\circ} \mathrm{C}$ por $4 \mathrm{~h}$. Segundo a literatura ${ }^{8,9}$, as dextranas pré-secas não podem ser usadas para preparo de soluções-estoque devido à possibilidade de retrogradação e redução da solubilidade. Desta forma, a secagem seria aplicada apenas para cálculo do teor de água nas dextranas. As soluções padrão seriam preparadas pesando-se as dextranas não secas, com a posterior correção do valor de sua concentração em função da sua umidade ${ }^{8}$.

Com o objetivo de verificar variação no teor de água em dextranas, em função da temperatura de aquecimento, foram realizadas análises termogravimétricas para amostras de dextranas com massa molecular entre $1,0 \times 10^{4}$ a $2,0 \times 10^{6}$ Daltons.

As curvas termogravimétricas obtidas apresentaram comportamento semelhante ao da Figura 1. Estas indicam duas regiões onde ocorrem perdas de massa: a primeira de 25 a $160{ }^{\circ} \mathrm{C}$ e a segunda de 280 a $450{ }^{\circ} \mathrm{C}$ (Tabela 1). A partir de $160{ }^{\circ} \mathrm{C}$ observa-se a existência de um patamar, que se mantém até $300{ }^{\circ} \mathrm{C}$; acima desta temperatura ocorre variação de massa, indicando a decomposição da amostra (Figura 1).

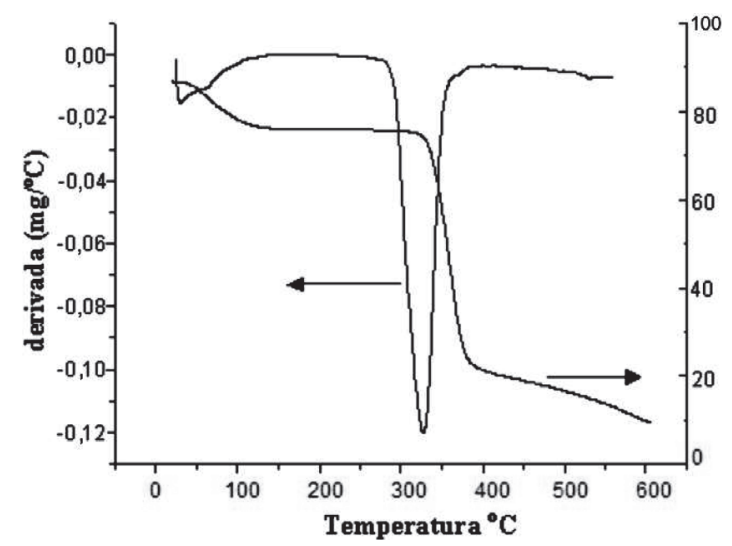

Figura 1. Termograma típico para as dextranas estudadas: $8 \mathrm{mg}$ da dextrana $T_{2000}, \mathrm{Ti}=20{ }^{\circ} \mathrm{C}$, razão de aquecimento de $20^{\circ} \mathrm{C} \mathrm{min} \mathrm{m}^{-1}$ até $600{ }^{\circ} \mathrm{C}$, fluxo de nitrogênio

Nos testes isotérmicos efetuados a 105 e $160{ }^{\circ} \mathrm{C}$ por um período de 3 h, observou-se que após 1 h de aquecimento a perda média de água nas dextranas se estabilizou em aproximadamente $10 \%$.

Na Tabela 1 , confrontando-se os valores a 105 e $160{ }^{\circ} \mathrm{C}$, nota-se que o aquecimento a $105{ }^{\circ} \mathrm{C}$ acarreta uma eliminação incompleta da água nos padrões $\mathrm{T}_{10}, \mathrm{~T}_{40}, \mathrm{~T}_{50}, \mathrm{~T}_{70}, \mathrm{~T}_{500} \mathrm{e}_{2000}$, respectivamente, de 2,5; 2,$9 ; 2,4 ; 3,4 ; 2,5$ e $2,4 \%$. Isto ocasionaria um erro sistemático, levando a uma quantificação do teor de dextranas totais inferior ao teor real.

Tabela 1. Perda de massa e transição vítrea (Tg) em padrões de dextrana observada experimentalmente em função da temperatura

\begin{tabular}{lccc}
\hline $\begin{array}{l}\text { Dextranas } \\
\text { (Daltons) }\end{array}$ & $\begin{array}{c}\text { Perda de } \\
\text { massa }(\%) \\
105{ }^{\circ} \mathrm{C}^{\mathrm{A}}\end{array}$ & $\begin{array}{c}\text { Perda de } \\
\text { massa }(\%) \\
160{ }^{\circ} \mathrm{C}^{\mathrm{B}}\end{array}$ & $\mathrm{T}_{\mathrm{g}}{ }^{\circ} \mathrm{C}$ \\
\hline $\mathrm{T} 10\left(1,0 \times 10^{4}\right)$ & $10,5 \%$ & $13,0 \%$ & - \\
$\mathrm{T} 40\left(4,0 \times 10^{4}\right)$ & $11,2 \%$ & $14,1 \%$ & 57,1 \\
$\mathrm{~T} 50\left(5,0 \times 10^{4}\right)$ & $6,5 \%$ & $8,9 \%$ & - \\
$\mathrm{T} 70\left(7,0 \times 10^{4}\right)$ & $9,8 \%$ & $13,2 \%$ & 57,0 \\
$\mathrm{~T} 500\left(5,0 \times 10^{5}\right)$ & $7,9 \%$ & $10,4 \%$ & 56,7 \\
$\mathrm{~T} 2000\left(2,0 \times 10^{6}\right)$ & $11,2 \%$ & $13,6 \%$ & 62,9 \\
\hline
\end{tabular}

A) Incerteza de $\pm 1,2 \%$

B) Incerteza de $\pm 1,0 \%$. 
A análise por calorimetria diferencial exploratória, DSC, realizada para a faixa de peso molecular das dextranas estudadas, demonstrou apenas uma transição endotérmica, em torno de $63{ }^{\circ} \mathrm{C}$ para a amostra $\mathrm{T}_{500}$, atribuída à transição vítrea $\left(\mathrm{T}_{\mathrm{g}}\right)$. Os valores de $\mathrm{T}_{\mathrm{g}}$ para as demais amostras estão mostrados na Tabela 1.

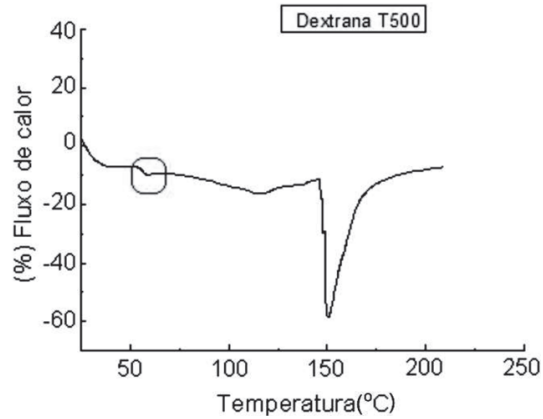

Figura 2. Termograma (DSC) típico para a dextrana T500: massa de 8,7 mg em atmosfera de nitrogênio com um fluxo de $20 \mathrm{~mL} \mathrm{~min}^{-1}$ e razão de aquecimento de $20^{\circ} \mathrm{C} \mathrm{min}^{-1}$ até $200{ }^{\circ} \mathrm{C}$

As análises por espectroscopia de absorção na região do infravermelho das amostras padrão de dextranas, antes e após o aquecimento, não apresentaram deslocamentos perceptíveis das bandas na região de $960-730 \mathrm{~cm}^{-1}$ característicos da ligação glicosídica, não fornecendo, portanto, evidências a respeito de alterações na estrutura molecular das dextranas ${ }^{13}$. Analogamente, os difratogramas de raio-X também não sugerirem alterações ${ }^{12}$ na estrutura cristalina das dextranas com o aquecimento a temperaturas de 70 e $200{ }^{\circ} \mathrm{C}$.

\section{Quantificações de dextranas totais em açúcares e bebidas}

A reprodutibilidade obtida nas análises de dextranas totais realizadas com padrões na faixa de peso molecular médio de $1,0 \mathrm{x}$ $10^{4}$ a 2,0 x $10^{6}$ Daltons variou de 2,6 a 4,4\% com média de 3,8\% e limites de detecção da ordem de $5,0 \times 10^{-2} \mathrm{mg} \mathrm{mL}^{-1}$.

A Tabela 2 reúne os resultados referentes ao teor de dextranas totais em açúcares.

Tabela 2. Teor de dextranas totais em açúcares comerciais

\begin{tabular}{cccc}
\hline Açúcar & Dextrana $(\mathrm{mg} / \mathrm{kg})$ & Açúcar & Dextrana $(\mathrm{mg} / \mathrm{kg})$ \\
\hline A01 & $4,75 \times 10^{2}$ & A14 & $1,00 \times 10^{3}$ \\
A02 & $1,26 \times 10^{3}$ & A15 & $1,10 \times 10^{2}$ \\
A03 & $2,88 \times 10^{2}$ & A16 & $2,30 \times 10^{2}$ \\
A04 & $6,95 \times 10^{2}$ & A17 & $1,29 \times 10^{3}$ \\
A05 & $6,09 \times 10^{2}$ & A18 & $1,16 \times 10^{3}$ \\
A06 & $4,34 \times 10^{2}$ & A19 & $8,20 \times 10^{2}$ \\
A07 & $1,27 \times 10^{3}$ & A20 & $1,25 \times 10^{3}$ \\
A08 & $7,39 \times 10^{2}$ & A21 & $1,13 \times 10^{3}$ \\
A09 & $1,63 \times 10^{3}$ & A22 & $1,09 \times 10^{3}$ \\
A10 & $4,75 \times 10^{2}$ & A23 & $1,04 \times 10^{3}$ \\
A11 & $3,89 \times 10^{2}$ & A24 & $1,16 \times 10^{3}$ \\
A12 & $3,64 \times 10^{2}$ & A25 & $1,66 \times 10^{3}$ \\
A13 & $2,37 \times 10^{2}$ & A26 & $1,84 \times 10^{3}$ \\
\hline
\end{tabular}

Segundo a Tabela 2, o teor médio de dextranas totais nos açúcares analisados foi de $870 \mathrm{mg} / \mathrm{kg}$, com mediana de $820 \mathrm{mg} / \mathrm{kg}$. Os valores de dextranas totais determinados nas amostras distribuemse por uma ampla faixa e são comparáveis com outros dados disponíveis na literatura ${ }^{2,5,8}$.

As Figuras 3 e 4 ilustram o acompanhamento da formação de flocos em uma das amostras de aguardente, em função da variação da concentração de dextranas totais em solução, da massa de precipitado e turbidez da amostra em função do tempo. As demais amostras apresentaram comportamento semelhante e conforme antecipado, a presença de dextranas é um fator extremamente importante para a formação de flocos em bebidas alcoólicas.

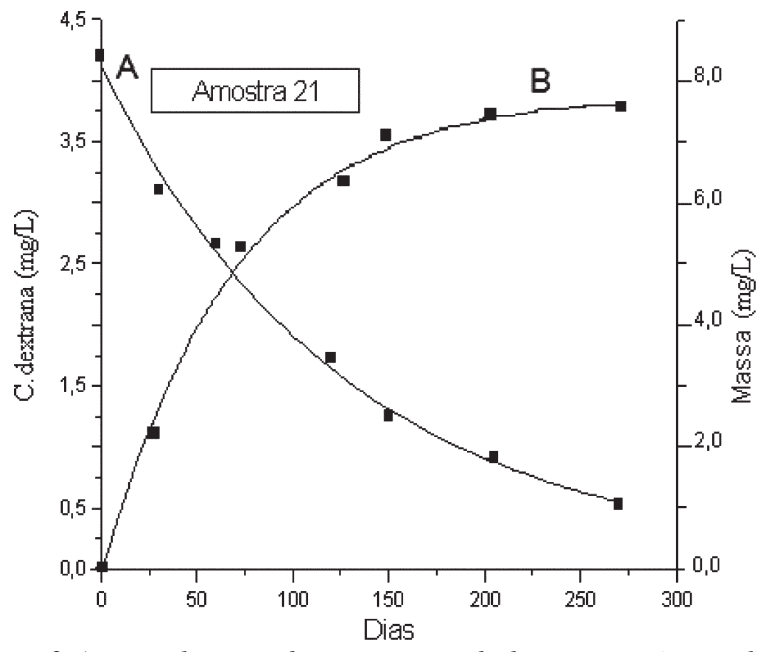

Figura 3. Acompanhamento da concentração de dextranas totais em solução (curva A) e da massa de precipitado formada (curva B) em função do tempo para uma amostra de aguardente 21

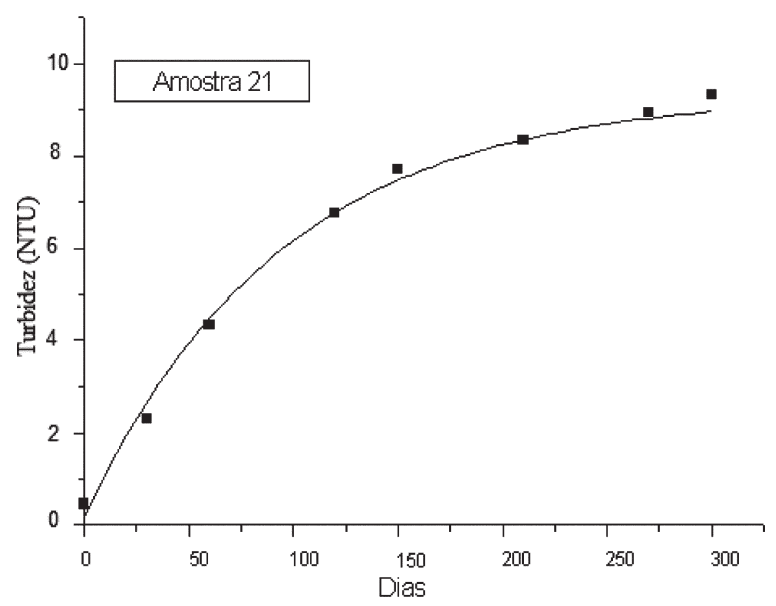

Figura 4. Acompanhamento da turbidez em função do tempo para uma amostra de aguardente 21

Nota-se que o teor de dextranas (curva A) diminui com o tempo até 275 dias de estocagem, quando a concentração de dextranas totais se encontra em torno de $0,5 \mathrm{mg} / \mathrm{L}$. A partir deste ponto, existe uma tendência para estabilização no valor do teor de dextranas e na massa do precipitado formado. Em concordância com as curvas A e B da Figura 3, a amostra apresentou um aumento de turbidez, com tendência à estabilidade a partir de 275 dias. Estes resultados em conjunto sugerem que a formação de precipitado na amostra em questão deixa de ser significativa a partir de 275 dias de estocagem.

Embora os açúcares analisados não tenham sido coletados junto aos produtores de cachaça, pode-se observar que o uso de açúcares, mesmo em quantidades de $6 \mathrm{~g} / \mathrm{L}^{6}$, com teor de dextranas em torno do valor mediano da Tabela 2 , transferirá para a cachaça uma quantidade de dextranas suficiente $(4,9 \mathrm{mg})$ para formação de precipitados em curto período de tempo.

Ainda com relação às Figuras 3 e 4, a amostra em questão apresenta um teor de dextranas da mesma ordem da mediana obti- 
Tabela 3. Teor de dextranas totais em cachaças adoçadas industriais, artesanais

\begin{tabular}{|c|c|c|c|c|c|c|c|}
\hline Cachaça & Dextrana $(\mathrm{mg} / \mathrm{L})$ & Cachaça & Dextrana (mg/L) & Cachaça & Dextrana $(\mathrm{mg} / \mathrm{L})$ & Cachaça & Dextrana $(\mathrm{mg} / \mathrm{L})$ \\
\hline CI 01 & 3,4 & CI 17 & 7,8 & CI 33 & 7,1 & CA 01 & 7,0 \\
\hline CI 02 & 1,9 & CI 18 & 6,0 & CI 34 & 6,3 & CA 02 & 4,0 \\
\hline CI 03 & 8,9 & CI 19 & 8,9 & CI 35 & 5,5 & CA 03 & 6,5 \\
\hline CI 04 & 4,5 & CI 20 & 5,6 & CI 36 & 7,5 & CA 04 & 2,3 \\
\hline CI 05 & 3,3 & CI 21 & 4,2 & CI 37 & 7,0 & CA 05 & 7,5 \\
\hline CI 06 & 8,7 & CI 22 & 5,0 & CI 38 & 6,0 & CA 06 & 11,2 \\
\hline CI 07 & 4,7 & CI 23 & 5,9 & CI 39 & 4,6 & CA 07 & 7,5 \\
\hline CI 08 & 3,1 & CI 24 & 9,0 & CI 40 & 6,8 & CA 08 & 5,8 \\
\hline CI 09 & 1,6 & CI 25 & 6,8 & CI 41 & 5,8 & CA 09 & 9,3 \\
\hline CI 10 & 3,2 & CI 26 & 7,5 & CI 42 & 7,8 & CA 10 & 7,8 \\
\hline CI 11 & 6,0 & CI 27 & 5,6 & CI 43 & 6,6 & CA 11 & 7,9 \\
\hline CI 12 & 4,5 & CI 28 & 3,3 & CI 44 & $<0,05$ & & \\
\hline CI 13 & 2,0 & CI 29 & 7,1 & CI 45 & 4,8 & & \\
\hline CI 14 & 8,7 & CI 30 & 5,3 & CI 46 & 2,3 & & \\
\hline CI 15 & 7,0 & CI 31 & 5,0 & & & & \\
\hline CI 16 & 5,4 & CI 32 & 5,1 & & & & \\
\hline
\end{tabular}

$\mathrm{CI}=$ Cachaça Industrial, CA = Cachaça Artesanal.

da para as cachaças industriais e artesanais contidas na Tabela 3, ou seja de 5,6 e 7,5 mg/L, respectivamente. Isso levará à turbidez e conseqüente formação de precipitados ao longo do tempo, no caso de teores mais elevados, pois a formação de flocos, pelo menos para o conjunto de amostras estudadas, cessa quando a concentração de dextranas em solução se torna inferior a $0,25 \mathrm{mg} / \mathrm{L}$.

Nota-se claramente pelas curvas das Figuras 3 e 4 que a formação de flocos em aguardentes é um processo cineticamente dependente. Portanto, uma simples filtração, mesmo à baixa temperatura, de uma bebida logo após a adição do açúcar pode não ser suficiente para contornar o problema.

Acrescente-se a isso o fato de que a formação de flocos não está restrita somente à presença de dextranas. A presença de outros carboidratos, polifenóis e aminoácidos é citada na literatura ${ }^{4,14-16}$ como componente de precipitados ou névoas em bebidas destiladas e fermentadas.

Um claro exemplo disto diz respeito ao aminoácido prolina, potencial formador de precipitados ou turbidez em bebidas contendo polifenóis ${ }^{17,18}$.

Em análises de cachaças adoçadas realizadas no Laboratório para o Desenvolvimento da Química da Aguardente (LDQA), a prolina apresentou-se como o segundo aminoácido mais abundante, com teores médios de $0,502 \mathrm{mg} / \mathrm{L}^{16}$. Em floco isolado de uma amostra de cachaça comercial adoçada, a percentagem em peso de prolina foi de $18,76 \%{ }^{16}$.

Assim, a formação de flocos em bebidas destiladas é um problema que está merecendo atenção de nosso Laboratório. Estudase a gênese dos flocos em soluções modelo contendo aminoácidos, dextranas e polifenóis avaliados em função de suas respectivas concentrações, da temperatura, da luz e do tempo de estocagem. Os resultados serão relatados futuramente.

\section{CONCLUSÕES}

Os resultados indicam que a secagem dos padrões de dextranas usados na elaboração de curvas de calibração pode ser efetuada a 105 ou $160{ }^{\circ} \mathrm{C}$. Testes isotérmicos indicam que nestas temperaturas o tempo de $4 \mathrm{~h}$ pode ser diminuído pela metade com a mesma eficiência na secagem das amostras.
Apesar dos açúcares analisados não serem os mesmos que foram utilizados pelos produtores de cachaças, percebe-se que a adição de açúcares, mesmo em valores que atendam à legislação, pode introduzir no produto dextranas em concentrações suficientes para desencadear o aparecimento de flocos e que a simples filtração após a adição de açúcar pode não ser suficiente para eliminar este problema.

\section{AGRADECIMENTOS}

Os autores agradecem a CAPES, CNPq e FAPESP pelo apoio financeiro

\section{REFERÊNCIAS}

1. Eggleston, G.; Food Chem. 2002, 78, 95.

2. Eggleston, G.; Legendre, B.; Tew, T.; Food Chem. 2004, 87, 119.

3. Tajchakavit. S.; Boye, J. I.; Bélanger, D.; Couture, R.; Food Res. Int. 2001, 34, 431.

4. Kruif. C. G.; Tuinier, R.; Food Hydrocolloids 2001, 15, 555.

5. Chistiane, F. B.; Peter, A. I.; J. Agric. Food Chem. 1992, 40, 227.

6. BRASIL. Instrução Normativa $n^{\circ} 13$, de 29 de junho de 2005, Ministério da Agricultura Pecuário e Abastecimento; Diário Oficial da União, seção 1 , p. 3-4, de 30/6/2005.

7. Rodrigues-Filho, M. G.; Dissertação de Mestrado, Universidade de São Paulo, Brasil, 2000.

8. Roberts, E. J.; Int. Sugar J. 1983, 85, 10.

9. Clarke, M. A.; Godshall M. A.; J. Assoc. Anal. Chem. 1988, 71, 276.

10. Sarkar, D.; Clarke, S. J.; Saska, M.; J. Am. Soc. Sugar Cane Technology 1990, 10, 122.

11. Leite, F.; Validação em Análise Química, Ed. Átomo: Campinas, 1996.

12. Kakudo, M.; Kasai, N.; X-Ray difraction by polymers. Elsivier Science Publishing Company: New York, 1972.

13. Colthup, N. B.; Daly, H.; Stepehen, E. N.; Introducton to Infrared and Raman. $3^{\text {rd }}$ ed., Boston Academic Press: Boston, 1990.

14. Freitas, V.; Carvalho, E.; Mateus, N.; Food Chem. 2003, 81, 503.

15. Refsgard, H. H. F.; Schaumburg, K.; Skibsted, L. H. Z.; Lebensm. Unters Forsch. 1996, 203, 287.

16. Boso, L. M.; Dissertação de Mestrado, Universidade de São Paulo, Brasil, 2001.

17. Siebert, K. J.; Carrasco, A.; Lynn, P. Y.; J. Agric. Food Chem. 1996, 44, 1997.

18. Siebert, K. J.; Troukhanova, N. V.; Lynn, P. Y.; J. Agric. Food Chem. 1996, $44,80$. 